\title{
Polynomial Approximation on Spheres - Generalizing de la Vallée-Poussin
}

\author{
Ian H. Sloan
}

\begin{abstract}
For trigonometric polynomial approximation on a circle, the century-old de la Vallée-Poussin construction has attractive features: it exhibits uniform convergence for all continuous functions as the degree of the trigonometric polynomial goes to infinity, yet it also has arbitrarily fast convergence for sufficiently smooth functions. This paper presents an explicit generalization of the de la Vallée-Poussin construction to higher dimensional spheres $\mathbb{S}^{d} \subseteq \mathbb{R}^{d+1}$. The generalization replaces the $C^{\infty}$ filter introduced by Rustamov by a piecewise polynomial of minimal degree. For the case of the circle the filter is piecewise linear, and recovers the de la Vallée-Poussin construction, while for the general sphere $\mathbb{S}^{d}$ the filter is a piecewise polynomial of degree $d$ and smoothness $C^{d-1}$. In all cases the approximation converges uniformly for all continuous functions, and has arbitrarily fast convergence for smooth functions.
\end{abstract}

2010 Mathematical subject classification: 42B99; 42C99.

Keywords: sphere; polynomial; approximation.

\section{Introduction}

We introduce in this paper a class of linear polynomial approximations on the unit sphere $\mathbb{S}^{d}=\left\{\mathbf{x} \in \mathbb{R}^{d+1}:\|\mathbf{x}\|_{\ell_{2}}=1\right\}$, which in the uniform norm

$$
\|f\|_{\infty}:=\sup _{\mathbf{x} \in \mathbb{S}^{d}}|f(\mathbf{x})|
$$

have the property of converging to $f$ for all continuous functions $f$, yet which exhibit arbitrarily fast convergence when $f$ is smooth.

In more detail, letting $\mathbb{P}_{L}\left(\mathbb{S}^{d}\right)$ denote the space of (spherical) polynomials of degree $\leqslant L$ (that is, the restriction to $\mathbb{S}^{d}$ of the polynomials in $\mathbb{R}^{d+1}$ of degree no more than $L$ ), for $f \in C\left(\mathbb{S}^{d}\right)$ we construct linear approximations $V_{L} f=V_{d, L} f$ in $\mathbb{P}_{2 L-1}\left(\mathbb{S}^{d}\right)$ that satisfy

$$
\left\|V_{L} f-f\right\|_{\infty} \leqslant c E_{L}(f)
$$

with $c$ independent of $L$ and $f$, where $E_{L}(f)$ is the error of best uniform polynomial approximation in $\mathbb{P}_{L}\left(\mathbb{S}^{d}\right)$,

$$
E_{L}(f):=\inf _{p \in \mathbb{P}_{L}\left(\mathbb{S}^{d}\right)}\|f-p\|_{\infty} .
$$

The approximation therefore inherits the best possible rates of uniform convergence, those given by the Jackson theorem for $\mathbb{S}^{d}$; see $[1,10]$. In particular, when $f$ is smooth the rate of convergence is arbitrarily fast. 
The construction used here relies on "delayed means", first introduced and exploited, in the context of 1-dimensional trigonometric series, by de la Vallée-Poussin. Stein [15] in 1957 showed, in a very general context, that uniformly bounded delayed means always exist if the degree is high enough. For polynomials on the sphere Rustamov [14] in 1993 showed, for all $L$, how to construct a uniformly bounded delayed-mean approximation. Rustamov's construction makes use of a $C^{\infty}$ function $h=h_{d}: \mathbb{R}^{+} \rightarrow \mathbb{R}^{+}$satisfying also

$$
h(x)=\left\{\begin{array}{l}
1 \text { for } x \in[0,1], \\
0 \text { for } x \in[2, \infty)
\end{array}\right.
$$

which is used to produce multipliers of the Fourier partial sum, in the sense that

$$
\sum_{\ell=0}^{L} \sum_{k=1}^{Z(d, \ell)} \widehat{f}_{\ell, k} Y_{\ell, k}(\mathbf{x})
$$

is replaced by

$$
\sum_{\ell=0}^{2 L} h\left(\frac{\ell}{L}\right) \sum_{k=1}^{Z(d, \ell)} \widehat{f}_{\ell, k} Y_{\ell, k}(\mathbf{x}) .
$$

Following a lead from signal analysis, we shall call a real-valued function with the property (1.3) a "filter function".

Rustamov's argument [14] relies on repeated summation by parts (see Lemma 4.1), together with the known positivity of the Cesàro means of order $d$. Related arguments have since been used in [9] and many other papers.

In this paper we devise spline filters $h$ for the sphere $\mathbb{S}^{d}$ of arbitrary dimension $d$, which are such that (1.1) and (1.4) both hold. For $d=1$ the construction recovers the de la Vallée-Poussin piecewise-linear filter.

The construction given in this paper is explicit if the Fourier coefficients of $f$ are available. In another paper [13] we will develop a related approximation scheme for the case in which only point values of $f$ are available.

The convergence property (1.1) implies, via the Weierstrass theorem, that

$$
\lim _{L \rightarrow \infty}\left\|V_{L} f-f\right\|_{\infty}=0 \quad \text { for all } \quad f \in C\left(\mathbb{S}^{d}\right)
$$

In turn this property implies, by the Banach-Steinhaus theorem (see, for example, [12] Theorem 5.3), that there exists $c_{d}>0$ independent of $L$ such that

$$
\left\|V_{L} f\right\|_{\infty} \leqslant c_{d}\|f\|_{\infty} .
$$

We know in advance that $V_{L}$ cannot be a projection onto the polynomial space $\mathbb{P}_{L}\left(\mathbb{S}^{d}\right)$, since the optimal linear projection $T_{L}: C\left(\mathbb{S}^{d}\right) \rightarrow \mathbb{P}_{L}\left(\mathbb{S}^{d}\right)$, in the sense of having the smallest value of the operator norm

$$
\left\|T_{L}\right\|:=\sup \left\{\left\|T_{L} f\right\|_{\infty}:\|f\|_{\infty} \leqslant 1\right\}
$$

is the $L_{2}$-orthogonal projection $\mathcal{P}_{L}$ onto $\mathbb{P}_{L}\left(\mathbb{S}^{d}\right)$, that is, the partial sum of the Fourier or Laplace series. This was proved for $d=1$ by Berman [3] and for general $d$ by Daugavet [4]. (For a lucid discussion see [12] Theorem 6.4.) Since the norm $\left\|\mathcal{P}_{L}\right\|$ of the orthogonal projection is known to grow unboundedly with $L$ for all $d \geqslant 1$, it is clear that no projection 
operator can be consistent with the property (1.4). Moreover, the operator $V_{L}$ cannot be positive, in the sense of mapping positive continuous functions to positive polynomials, since by results of Korovkin [8] the best possible rate of convergence (the "saturation order") for polynomial approximation by a positive operator is $L^{-2}$, which contradicts (1.1) for sufficiently smooth $f$.

An approximation satisfying (1.1) has long been known for $d=1$ : in that case the century-old construction of de la Vallée-Poussin (see [5] Chapter 9, Section 3), meets the requirements. That approximation is exact for polynomials of degree $\leqslant L$, a property that leads to high accuracy for large $L$; and on the other hand has uniformly bounded norm by virtue of being a simple linear combination of two different Cesàro means, which for $d=1$ are separately positive. We sketch the de la Vallée-Poussin construction in Section 2.

A very different generalization of the de la Vallée-Poussin approximation that again satisfies both (1.1) and (1.4) was introduced by Filbir and Themistoclakis in [6]. The construction in that paper relies on multiplication of two Fourier kernels, rather than of multiplication of a Fourier kernel by a filter function.

Both the present approximation and that of [6] are different from the de la Vallée-Poussin mean introduced by Berens and $\mathrm{Li}$ in [2]. The linear operator for that construction is a positive operator, and is now known (see [17]) to have a saturation order of $L^{-1}$. (The kernel for the de la Vallée-Poussin mean, replacing $H_{L}(\mathbf{x}, \mathbf{y})$ in (4.1) below, is a constant times $\left.(1+\mathbf{x} \cdot \mathbf{y})^{L} \cdot\right)$

After preliminaries in Section 3, we describe in Section 4 Rustamov's filtered approximation, taking care to keep separate the arguments that do not need high smoothness of the filter function $h$. In Section 5 the piecewise-polynomial construction of minimal smoothness and minimal degree is described, and shown to have the same properties as the $C^{\infty}$ version.

An interesting question not addressed in this paper is the dependence of $\sup \left\|V_{L}\right\|$ on $d$. For the operator constructed in [6] the supremum of the norm is of order $O\left(3^{d}\right)$. For the operator constructed in Section 5 the corresponding question is at this time still open.

\section{The de la Vallée-Poussin construction for $d=1$}

For an integrable function $f$ on the unit circle $\mathbb{T}$, the $n$th partial sum of the Fourier series of $f$ is

$$
\begin{aligned}
S_{n} f(\theta):=\frac{1}{2} a_{0} & +a_{1} \cos (\theta)+\cdots+a_{n} \cos (n \theta) \\
& +b_{1} \sin (\theta)+\cdots+b_{n} \sin (n \theta)
\end{aligned}
$$

with

$$
a_{j}=\frac{1}{\pi} \int_{0}^{2 \pi} f(\phi) \cos (j \phi) \mathrm{d} \phi, \quad b_{j}=\frac{1}{\pi} \int_{0}^{2 \pi} f(\phi) \sin (j \phi) \mathrm{d} \phi .
$$

It can be written, with the aid of trigonometric identities, as

$$
\begin{aligned}
S_{n} f(\theta) & =\frac{1}{\pi} \int_{0}^{2 \pi} f(\phi)\left[\frac{1}{2}+\cos (\theta-\phi)+\cos (2(\theta-\phi))+\cdots+\cos (n(\theta-\phi))\right] \mathrm{d} \phi \\
& =\frac{1}{\pi} \int_{0}^{2 \pi} f(\phi) D_{n}(\theta-\phi) \mathrm{d} \phi,
\end{aligned}
$$


where $D_{n}$ is the Dirichlet kernel (see [5], equation (1.9))

$$
D_{n}(\theta):=\frac{\sin ((2 n+1) \theta / 2)}{2 \sin (\theta / 2)}, \quad \theta \in \mathbb{R} .
$$

The partial sum $S_{n} f$ is of course the $L_{2}$-orthogonal projection of $f$ on the space of trigonometric polynomials of degree $\leqslant n$.

The de la Vallée-Poussin approximation to $f$ (see [5] Chapter 9, Section 3) is the "delayed" mean of $n$ successive partial sums,

$$
\begin{aligned}
V_{n} f(\theta) & =\frac{1}{n}\left[S_{n} f(\theta)+\cdots+S_{2 n-1} f(\theta)\right] \\
& =\frac{1}{\pi} \int_{0}^{2 \pi} f(\phi)\left(\sum_{j=0}^{2 n-1} h_{n, j} \cos (j(\theta-\phi))\right) \mathrm{d} \phi,
\end{aligned}
$$

where the prime on the sum indicates that the first term is to be halved, and

$$
h_{n, j}= \begin{cases}1 & \text { for } \quad 0 \leqslant j \leqslant n \\ 1-\frac{j-n}{n} & \text { for } n+1 \leqslant j \leqslant 2 n-1 .\end{cases}
$$

It is easily seen that

$$
V_{n} f(\theta)=2 C_{2 n-1} f(\theta)-C_{n-1} f(\theta),
$$

where $C_{m} f$ for $m \geqslant 0$ is the (first) Cesàro mean of $f$,

$$
\begin{aligned}
C_{m} f(\theta) & =\frac{1}{m+1}\left[S_{0} f(\theta)+\cdots+S_{m} f(\theta)\right] \\
& =\frac{1}{\pi} \int_{0}^{2 \pi} f(\phi) \frac{1}{m+1}\left[D_{0}(\theta-\phi)+D_{1}(\theta-\phi)+\cdots+D_{m}(\theta-\phi)\right] \mathrm{d} \phi \\
& =\frac{1}{\pi} \int_{0}^{2 \pi} f(\phi) F_{m}(\theta-\phi) \mathrm{d} \phi,
\end{aligned}
$$

with $F_{m}$ being the Fejér kernel (see [5], p. 3)

$$
F_{m}(\theta)=\frac{1}{2(m+1)} \frac{\sin ^{2}((m+1) \theta / 2)}{\sin ^{2}(\theta / 2)},
$$

which is manifestly non-negative. It then follows from (2.3) and (2.4) and the non-negativity of $F_{m}$ that

$$
\begin{aligned}
\left|V_{n} f(\theta)\right| & =\left|\frac{1}{\pi} \int_{0}^{2 \pi} f(\phi)\left[2 F_{2 n-1}(\theta-\phi)-F_{n-1}(\theta-\phi)\right] \mathrm{d} \phi\right| \\
& \leqslant\|f\|_{\infty} \frac{1}{\pi} \int_{0}^{2 \pi}\left[2 F_{2 n-1}(\theta-\phi)+F_{n-1}(\theta-\phi)\right] \mathrm{d} \phi \\
& =\|f\|_{\infty}(2+1),
\end{aligned}
$$

giving the uniform bound

$$
\left\|V_{n}\right\| \leqslant 3
$$


From (2.1), or from $(2.2)$ and the fact that $h_{n, j}=1$ for $0 \leqslant j \leqslant n$, it follows that

$$
V_{n} p=p \quad \text { for all } \quad p \in \mathbb{P}_{n}(\mathbb{T})
$$

where $\mathbb{P}_{n}(\mathbb{T})$ is the space of trigonometric polynomials of degree $\leqslant n$. In turn this leads to

$$
\begin{aligned}
\left\|V_{n} f-f\right\|_{\infty} & =\left\|V_{n}(f-p)-(f-p)\right\|_{\infty} \\
& \leqslant\left(1+\left\|V_{n}\right\|\right)\|f-p\|_{\infty}
\end{aligned}
$$

for all $p \in \mathbb{P}_{n}(\mathbb{T})$, and hence by a judicious choice of $p$ and the use of (2.5),

$$
\left\|V_{n} f-f\right\|_{\infty} \leqslant(1+3) E_{n}(f)=4 E_{n}(f) .
$$

\section{Preliminaries}

The space $L_{2}=L_{2}\left(\mathbb{S}^{d}\right)$ is the usual Hilbert space of square-integrable functions on $\mathbb{S}^{d}$ with the inner product

$$
(f, g)_{L_{2}}:=\int_{\mathbb{S}^{d}} f(\mathbf{x}) g(\mathbf{x}) \mathrm{d} \omega(\mathbf{x})
$$

and the norm $\|f\|_{L_{2}}:=\sqrt{(f, f)_{L_{2}}}$. Here $\omega=\omega_{d}$ denotes the (Lebesgue) surface measure on $\mathbb{S}^{d}$. The space of continuous functions on $\mathbb{S}^{d}$ is denoted by $C=C\left(\mathbb{S}^{d}\right)$ and is a Banach space with the supremum norm

$$
\|f\|_{\infty}:=\sup _{\mathbf{x} \in \mathbb{S}^{d}}|f(\mathbf{x})|
$$

The space $\mathbb{P}_{L}=\mathbb{P}_{L}\left(\mathbb{S}^{d}\right)$ of all spherical polynomials on $\mathbb{S}^{d}$ of degree $\leqslant L$ contains the restrictions to $\mathbb{S}^{d}$ of all polynomials on $\mathbb{R}^{d+1}$ of degree $\leqslant L$. The dimension of $\mathbb{P}_{L}$ is given by

$$
d_{L}=\operatorname{dim}\left(\mathbb{P}_{L}\right)=\frac{(2 L+d) \Gamma(L+d)}{\Gamma(d+1) \Gamma(L+1)} .
$$

A spherical harmonic of degree $\ell \in \mathbb{N}_{0}$ is the restriction to $\mathbb{S}^{d}$ of a harmonic homogeneous polynomial of degree $\ell$ on $\mathbb{R}^{d+1}$. The space $\mathbb{H}_{\ell}=\mathbb{H}_{\ell}\left(\mathbb{S}^{d}\right)$ of spherical harmonics of degree $\ell \in \mathbb{N}_{0}$ (together with the zero function) has the dimension $Z(d, \ell)=\operatorname{dim}\left(\mathbb{H}_{\ell}\right)$, given by

$$
Z(d, 0)=1 ; \quad Z(d, \ell)=\frac{(2 \ell+d-1) \Gamma(\ell+d-1)}{\Gamma(d) \Gamma(\ell+1)}, \quad \ell \in \mathbb{N}
$$

In this paper, for any $\ell \in \mathbb{N}_{0}$, the set

$$
\left\{Y_{\ell, k}: k=1,2, \ldots, Z(d, \ell)\right\}
$$

denotes a real $L_{2}$-orthonormal basis of $\mathbb{H}_{\ell}$. Furthermore, $\mathbb{P}_{L}=\bigoplus_{\ell=0}^{L} \mathbb{H}_{\ell}$, and the union over $\ell=0,1, \ldots, L$ of the sets (3.1) forms an $L_{2}$-orthonormal basis of $\mathbb{P}_{L}$.

The spherical harmonics on $\mathbb{S}^{d}$ of degree $\ell$ satisfy the addition theorem: for any $L_{2^{-}}$ orthonormal basis (3.1) of $\mathbb{H}_{\ell}$ we have, with $\tau=\tau_{d}=d / 2-1$,

$$
\sum_{k=1}^{Z(d, \ell)} Y_{\ell, k}(\mathbf{x}) Y_{\ell, k}(\mathbf{y})=\frac{Z(d, \ell)}{\left|\mathbb{S}^{d}\right|} \frac{P_{\ell}^{(\tau, \tau)}(\mathbf{x} \cdot \mathbf{y})}{P_{\ell}^{(\tau, \tau)}(1)}
$$


where $P_{\ell}^{(\alpha, \beta)}$ is the Jacobi polynomial of degree $\ell$, and $\left|\mathbb{S}^{d}\right|$ is the total surface measure of $\mathbb{S}^{d}$.

The kernel $K_{\ell}=K_{d, \ell}$ defined by

$$
K_{\ell}(\mathbf{x}, \mathbf{y})=K_{\ell}(\mathbf{x} \cdot \mathbf{y})=\sum_{k=1}^{Z(d, \ell)} Y_{\ell, k}(\mathbf{x}) Y_{\ell, k}(\mathbf{y})=\frac{Z(d, \ell)}{\left|\mathbb{S}^{d}\right|} \frac{P_{\ell}^{(\tau, \tau)}(\mathbf{x} \cdot \mathbf{y})}{P_{\ell}^{(\tau, \tau)}(1)}
$$

is the reproducing kernel in the space $\mathbb{H}_{\ell}$, in the sense that

$$
\left(p, K_{\ell}(\mathbf{x}, \cdot)\right)_{L_{2}}=p(\mathbf{x}) \text { for all } p \in \mathbb{H}_{\ell}, \mathbf{x} \in \mathbb{S}^{d} .
$$

It will play an important role in the following section.

The union over all $\ell \in \mathbb{N}_{0}$ of the $L_{2}$-orthonormal bases (3.1) of $\mathbb{H}_{\ell}$ forms a complete orthonormal system for $L_{2}$. Thus any function $f \in L_{2}$ can be expanded into a Fourier series (or Laplace series) with respect to this orthonormal system: in the $L_{2}$ sense

$$
f=\sum_{\ell=0}^{\infty} \sum_{k=1}^{Z(d, \ell)} \widehat{f}_{\ell, k} Y_{\ell, k}
$$

where the Fourier coefficients are given by

$$
\widehat{f}_{\ell, k}=\left(f, Y_{\ell, k}\right)_{L_{2}}=\int_{\mathbb{S}^{d}} f(\mathbf{x}) Y_{\ell, k}(\mathbf{x}) \mathrm{d} \omega(\mathbf{x}) .
$$

Finally, we recall the formula for summation by parts, or Abel transformation. Given the series

$$
\sum_{i=0}^{m} a_{i} f_{i}
$$

where $a_{i}$ and $f_{i}$ are real numbers, the summation by parts formula, easily verified, is

$$
\sum_{i=0}^{m} a_{i} f_{i}=a_{m} F_{m}-\sum_{i=0}^{m-1} \Delta a_{i} F_{i}
$$

where

$$
F_{i}=\sum_{j=0}^{i} f_{j}
$$

and $\Delta a_{i}$ is the forward difference of the sequence $\left(a_{i}\right)$,

$$
\Delta a_{i}=a_{i+1}-a_{i}, \quad i=0, \cdots, m-1 .
$$

\section{The filtered approximation scheme}

Given $f \in L_{2}\left(\mathbb{S}^{d}\right)$, the approximation takes the form

$$
V_{L} f(\mathbf{x})=\left(f, H_{L}(\mathbf{x}, \cdot)\right)_{L_{2}}=\int_{\mathbb{S}^{d}} f(\mathbf{y}) H_{L}(\mathbf{x}, \mathbf{y}) \mathrm{d} \omega(\mathbf{y}),
$$

where $H_{L}(\mathbf{x}, \mathbf{y}):=H_{L}(\mathbf{x} \cdot \mathbf{y}):=H_{d, L}(\mathbf{x} \cdot \mathbf{y})$, and

$$
H_{L}(t)=\sum_{\ell=0}^{2 L} h\left(\frac{\ell}{L}\right) K_{\ell}(t), \quad t \in[-1,1],
$$


with $K_{\ell}$ the reproducing kernel in $\mathbb{H}_{\ell}$ defined in (3.3); and $h: \mathbb{R}^{+} \rightarrow \mathbb{R}^{+}$satisfies (1.3), together with further properties to be developed below. It is useful to rewrite the approximation, using (4.2) and (3.3), as

$$
V_{L} f(\mathbf{x})=\sum_{\ell=0}^{2 L} h\left(\frac{\ell}{L}\right) \sum_{k=1}^{Z(d, \ell)} \widehat{f}_{\ell, k} Y_{\ell, k}(\mathbf{x})
$$

In this form it is clear that $V_{L} f$ is a filtered version of the Fourier series partial sum

$$
\sum_{\ell=0}^{2 L} \sum_{k=1}^{Z(d, \ell)} \widehat{f}_{\ell, k} Y_{\ell, k}(\mathbf{x})
$$

It is also clear from (1.3) that $V_{L}$ reproduces polynomials of degree up to $L$, in the sense that

$$
V_{L} p=p \quad \text { for all } p \in \mathbb{P}_{L}
$$

The properties of the polynomial approximation $V_{L}$ depend strongly on the nature of the filter $h$. We recover the orthogonal approximation $T_{L} f$ by setting $h(x)=0$ for $x>1$, but smoother filter functions have a certain advantage: namely that under certain conditions on $h, V_{L}$ is uniformly bounded in the uniform norm. The following result is implied by [14], Lemma 3.1.

Proposition 4.1. Let $V_{L}$ be the linear operator defined by (4.1) and (4.2), where $h$ : $\mathbb{R}^{+} \rightarrow \mathbb{R}^{+}$satisfies (1.3). Assume there exist $L_{d} \geqslant 0$ and $C_{d}>0$ such that for $L \geqslant L_{d}$ we have

$$
\sum_{\ell=0}^{2 L-1}\left|\Delta^{d+1} h\left(\frac{\ell}{L}\right)\right| \leqslant \frac{C_{d}}{L^{d}}
$$

where $\Delta^{d+1} h\left(\frac{\ell}{L}\right)$ denotes the $(d+1)$ st forward difference of the sequence $h(0 / L), h(1 / L)$, $h(2 / L), \ldots$ Then

$$
\sup _{L \geqslant 0}\left\|V_{L}\right\|<\infty
$$

The proposition rests upon the following simple lemma.

Lemma 4.1. The kernel $H_{L}$ defined by (4.2) and (3.3) can be expressed as

$$
H_{L}(t)=(-1)^{d+1} \sum_{\ell=0}^{2 L-1}\left(\begin{array}{c}
\ell+d \\
d
\end{array}\right)\left(\Delta^{d+1} h\left(\frac{\ell}{L}\right)\right) M_{\ell}^{(d)}(t), \quad t \in[-1,1],
$$

where $M_{\ell}^{(d)}(t)$ is the dth Cesàro mean of the kernels $K_{\ell}(t)$, defined below by (4.7), (4.8), (4.9) and (4.10).

Proof. Given the sequence $K_{0}(t), K_{1}(t), \ldots$, the $\ell$ th partial sum $Z_{\ell}^{(0)}$ and the successive Cesàro sums are defined, for $t \in[-1,1]$, by (see [12], Section 6.7)

$$
\begin{aligned}
& Z_{\ell}^{(0)}(t)=K_{0}(t)+\ldots+K_{\ell}(t) \\
& Z_{\ell}^{(n)}(t)=Z_{0}^{(n-1)}(t)+\ldots+Z_{\ell}^{(n-1)}(t) \text { for } n \geqslant 1 .
\end{aligned}
$$


The corresponding Cesàro means are then given by

$$
M_{\ell}^{(n)}(t)=\frac{1}{c_{\ell}^{(n)}} Z_{\ell}^{(n)}(t), \quad n \geqslant 0, \ell \geqslant 0
$$

where

$$
c_{\ell}^{(n)}=\left(\begin{array}{c}
\ell+n \\
n
\end{array}\right)
$$

It is clear that $M_{\ell}^{(n)}(t)$ can be written as a linear combination of $K_{i}(t)$ for $0 \leqslant i \leqslant \ell$. The above choice of $c_{\ell}^{(n)}$ ensures that the coefficient of $K_{0}(t)$ is always 1 , that is

$$
M_{\ell}^{(n)}(t)=K_{0}(t)+a_{\ell, 1}^{(n)} K_{1}(t)+\ldots+a_{\ell, \ell}^{(n)} K_{\ell}(t) \quad \text { for } 0 \leqslant \ell \leqslant L
$$

for some positive numbers $a_{\ell, i}^{(n)}$.

Application of the summation by parts formula (3.4) to the formally infinite series

$$
H_{L}(t)=\sum_{\ell=0}^{\infty} h\left(\frac{\ell}{L}\right) K_{\ell}(t)
$$

yields

$$
H_{L}(t)=-\sum_{\ell=0}^{\infty}\left(\Delta h\left(\frac{\ell}{L}\right)\right) Z_{\ell}^{(0)}(t),
$$

and summation by parts a further $d$ times yields in turn

$$
\begin{aligned}
H_{L}(t) & =(-1)^{d+1} \sum_{\ell=0}^{\infty}\left(\Delta^{d+1} h\left(\frac{\ell}{L}\right)\right) Z_{\ell}^{(d)}(t) \\
& =(-1)^{d+1} \sum_{\ell=0}^{2 L-1}\left(\begin{array}{c}
\ell+d \\
d
\end{array}\right)\left(\Delta^{d+1} h\left(\frac{\ell}{L}\right)\right) M_{\ell}^{(d)}(t),
\end{aligned}
$$

where in the last step we used (4.9) and (4.10) and the fact that all forward differences of $h\left(\frac{\ell}{L}\right)$ vanish for $\ell \geqslant 2 L$.

Proof of Proposition 4.1. $\quad$ It is easily seen from (4.1) that

$$
\left\|V_{L}\right\|=\sup _{\mathbf{x} \in \mathbb{S}^{d}} \int_{\mathbb{S}^{d}}\left|H_{L}(\mathbf{x} \cdot \mathbf{y})\right| \mathrm{d} \omega(\mathbf{y})=\int_{\mathbb{S}^{d}}\left|H_{L}(\mathbf{z} \cdot \mathbf{y})\right| \mathrm{d} \omega(\mathbf{y}), \quad \mathbf{z} \in \mathbb{S}^{d}
$$

where the second equality follows from the fact that the last integral is independent of $\mathbf{z}$.

It follows from Lemma 4.1 that

$$
\begin{aligned}
\int_{\mathbb{S}^{d}}\left|H_{L}(\mathbf{z} \cdot \mathbf{y})\right| \mathrm{d} \omega(\mathbf{y}) & =\int_{\mathbb{S}^{d}}\left|\sum_{\ell=0}^{2 L-1}\left(\begin{array}{c}
\ell+d \\
d
\end{array}\right)\left(\Delta^{d+1} h\left(\frac{\ell}{L}\right)\right) M_{\ell}^{(d)}(\mathbf{z} \cdot \mathbf{y})\right| \mathrm{d} \omega(\mathbf{y}) \\
& \leqslant \int_{\mathbb{S}^{d}} \sum_{\ell=0}^{2 L-1}\left(\begin{array}{c}
\ell+d \\
d
\end{array}\right)\left|\Delta^{d+1} h\left(\frac{\ell}{L}\right)\right| M_{\ell}^{(d)}(\mathbf{z} \cdot \mathbf{y}) \mathrm{d} \omega(\mathbf{y}),
\end{aligned}
$$


where in the last step we used the crucial fact, due to Kogbetliantz [7], and subsequently proved more simply by Reimer [11], that the $d$ th Cesàro mean on $\mathbb{S}^{d}$ is non-negative. Now we use, from (4.11),

$$
\int_{\mathbb{S}^{d}} M_{\ell}^{(d)}(\mathbf{z} \cdot \mathbf{y}) \mathrm{d} \omega(\mathbf{y})=\int_{\mathbb{S}^{d}} K_{0}(\mathbf{z} \cdot \mathbf{y}) \mathrm{d} \omega(\mathbf{y})=1, \quad \mathbf{z} \in \mathbb{S}^{d}, \ell \geqslant 0 .
$$

It then follows, using (4.12), that

$$
\left\|V_{L}\right\|=\int_{\mathbb{S}^{d}}\left|H_{L}(\mathbf{z} \cdot \mathbf{y})\right| \mathrm{d} \omega(\mathbf{y}) \leqslant \sum_{\ell=0}^{2 L-1} \frac{(\ell+d) \ldots(\ell+1)}{d !}\left|\Delta^{d+1} h\left(\frac{\ell}{L}\right)\right|,
$$

and for $L \geqslant d$ that

$$
\left\|V_{L}\right\| \leqslant \frac{(3 L)^{d}}{d !} \sum_{\ell=0}^{2 L-1}\left|\Delta^{d+1} h\left(\frac{\ell}{L}\right)\right|
$$

Using now the assumption (4.5), we obtain

$$
\left\|V_{L}\right\| \leqslant \frac{3^{d}}{d !} C_{d} \quad \text { for } L \geqslant d .
$$

Since $\left\|V_{L}\right\|$ is finite also for the exceptional values $L=0,1, \ldots, d-1$, the desired bound holds for all $L$. The proof of Proposition 4.1 is therefore complete.

The following corollary ensures that, under the conditions in Proposition 4.1, $V_{L} f$ converges to $f$ with at least the rate $E_{L}(f)$.

Corollary 4.1. Let $V_{L}$ and $M_{d}$ be defined as in Proposition 4.1. If (4.5) holds, then for $f \in C\left(\mathbb{S}^{d}\right)$ there holds

$$
\left\|V_{L} f-f\right\|_{\infty} \leqslant\left(\sup _{L \geqslant 0}\left\|V_{L}\right\|+1\right) E_{L}(f),
$$

where $E_{L}(f)$ is the error of best approximation defined by (1.2).

Proof. Because of the linearity of $V_{L}$ and the property (4.4), for all $p \in \mathbb{P}_{L}\left(\mathbb{S}^{d}\right)$ we have

$$
V_{L} f-f=V_{L}(f-p)-(f-p),
$$

and hence, on setting $M_{d}:=\sup _{L \geqslant 0}\left\|V_{L}\right\|$,

$$
\left\|V_{L} f-f\right\|_{\infty} \leqslant\left(\left\|V_{L}\right\|+1\right)\|f-p\|_{\infty} \leqslant\left(M_{d}+1\right)\|f-p\|_{\infty} .
$$

By making an optimal choice of $p$ we then obtain

$$
\left\|V_{L} f-f\right\|_{\infty} \leqslant\left(M_{d}+1\right) E_{L}(f),
$$

completing the proof.

The next corollary is stated by Rustamov in Lemma 3.1 of [14] under the assumption that $h \in C^{\infty}$.

Corollary 4.2. Let $V_{L}$ be defined as in Proposition 4.1. Assume $h \in C^{d+1}\left(\mathbb{R}^{+}\right)$. Then $\left\|V_{L}\right\|$ is bounded uniformly in $L$. 
Proof. For a function $g \in C^{d+1}\left(\mathbb{R}^{+}\right)$a well known result in the theory of finite differences (following from the mean value theorem) is

$$
\left|\Delta^{d+1} g(\ell)\right| \leqslant \sup _{z \in \mathbb{R}^{+}}\left|g^{(d+1)}(z)\right|
$$

In the present application we set $g(z)=h(z / L)$ and use the chain rule for differentiation to obtain, for $L \geqslant 1$,

$$
\left|\Delta^{d+1} h\left(\frac{\ell}{L}\right)\right| \leqslant \frac{1}{L^{d+1}} \max _{x \in[0,2]}\left|h^{(d+1)}(x)\right|,
$$

where we used also the fact that $\operatorname{supp} h=[0,2]$. Thus for $L \geqslant 1$ we have

$$
\sum_{\ell=0}^{2 L-1}\left|\Delta^{d+1} h\left(\frac{\ell}{L}\right)\right| \leqslant \frac{2 L}{L^{d+1}}\left\|h^{(d+1)}\right\|_{\infty}=\frac{2\left\|h^{(d+1)}\right\|_{\infty}}{L^{d}},
$$

which satisfies the sufficient condition (4.5) with $C_{d}=2\left\|h^{(d+1)}\right\|_{\infty}$.

The smoothness requirement in this corollary for $d=1$, namely $h \in C^{2}$, excludes by a wide margin the merely $C^{0}$ filter function used by de la Vallée-Poussin for $d=1$. In the next section we construct and justify a less smooth piecewise-polynomial filter, which may be reasonably regarded as the natural extension of the de la Vallée-Poussin construction to general $d$.

\section{Generalizing de la Vallée-Poussin}

Here we show how to construct spline filters $h$ for use in $V_{L} f$, filters that satisfy the condition in Proposition 4.1 but not the condition in Corollary 4.2. These filters belong not to $C^{d+1}\left(\mathbb{R}^{+}\right)$, but rather to $C^{d-1}\left(\mathbb{R}^{+}\right)$. The construction reduces to the de la Vallée-Poussin filter for $d=1$.

\section{The construction}

Partition the interval [1,2] into $d$ equal subintervals. Define $h \in C^{d-1}\left(\mathbb{R}^{+}\right)$to be the unique piecewise polynomial of degree no less than $d$ that satisfies (1.3) and in addition

$$
\left.h\right|_{\left[1+\frac{j-1}{d}, 1+\frac{j}{d}\right]} \in \mathbb{P}_{d}, \quad j=1, \ldots, d,
$$

where $\mathbb{P}_{d}$ is the space of polynomials on the real line of degree at most $d$. (Existence and uniqueness follow from the well known properties of polynomial splines with simple boundary conditions.)

Theorem 5.1. For $d \geqslant 1$, let $h \in C^{d-1}\left(\mathbb{R}^{+}\right)$be the unique piecewise polynomial constructed as above, and let $V_{L}$ be the linear operator defined by (4.1) and (4.2). Then

$$
\sup _{L \geqslant 0}\left\|V_{L}\right\|<\infty
$$

and there exists $c>0$ such that

$$
\left\|V_{L} f-f\right\|_{\infty} \leqslant c E_{L}(f), \quad \text { for } f \in C\left(\mathbb{S}^{d}\right) .
$$

The result follows from Proposition 4.1 and Corollary 4.1. It would be perhaps tedious to prove the general case. More usefully, in the following we demonstrate the proof of the theorem for $d=1$, for which the construction is exactly that of de la Vallée-Poussin, and $d=2$, which is undoubtedly the most important extension. After that we comment on the the general case, whose truth will by then be clear. 


\subsection{The case $d=1$}

For $d=1$ we have

$$
h(x)=\left\{\begin{array}{l}
1 \quad \text { for } x \in[0,1] \\
2-x \quad \text { for } x \in[1,2] \\
0 \text { for } x \in[2, \infty)
\end{array}\right.
$$

We easily find

$$
\Delta h\left(\frac{\ell}{L}\right)=\left\{\begin{array}{l}
0 \quad \text { for } 0 \leqslant \ell \leqslant L-1 \\
-\frac{1}{L} \quad \text { for } L \leqslant \ell \leqslant 2 L-1
\end{array}\right.
$$

and hence

$$
\Delta^{2} h\left(\frac{\ell}{L}\right)=\left\{\begin{array}{l}
0 \quad \text { for } 0 \leqslant \ell \leqslant L-2 \\
-\frac{1}{L} \quad \text { for } \ell=L-1 \\
0 \quad \text { for } L \leqslant \ell \leqslant 2 L-2 \\
+\frac{1}{L} \quad \text { for } \ell=2 L-1
\end{array}\right.
$$

giving

$$
\sum_{\ell=0}^{2 L-1}\left|\Delta^{2} h\left(\frac{\ell}{L}\right)\right|=\frac{2}{L},
$$

which satisfies (4.5) for $d=1$ with $C_{1}=2$. Thus for this construction of $h$ the condition in Proposition 4.1 is satisfied.

\subsection{The case $d=2$}

In this case we have explicitly

$$
h(x)=\left\{\begin{array}{l}
1 \quad \text { for } x \in[0,1] \\
1-2(x-1)^{2} \text { for } x \in\left[1, \frac{3}{2}\right] \\
2(2-x)^{2} \text { for } x \in\left[\frac{3}{2}, 2\right] \\
0 \text { for } x \in[2, \infty)
\end{array}\right.
$$

We easily find, for $L$ even and $L \geqslant 2$,

$$
\Delta^{2} h\left(\frac{\ell}{L}\right)=\left\{\begin{array}{l}
0 \quad \text { for } 0 \leqslant \ell \leqslant L-2 \\
-\frac{2}{L^{2}} \quad \text { for } \ell=L-1 \\
-\frac{4}{L^{2}} \quad \text { for } L \leqslant \ell \leqslant \frac{3}{2} L-2 \\
0 \quad \text { for } \ell=\frac{3}{2} L-1 \\
\frac{4}{L^{2}} \quad \text { for } \frac{3}{2} L \leqslant \ell \leqslant 2 L-2 \\
\frac{2}{L^{2}} \quad \text { for } \ell=2 L-1
\end{array}\right.
$$

and hence

$$
\Delta^{3} h\left(\frac{\ell}{L}\right)=\left\{\begin{array}{l}
0 \quad \text { for } 0 \leqslant \ell \leqslant L-3 \\
-\frac{2}{L^{2}} \quad \text { for } L-2 \leqslant \ell \leqslant L-1 \\
0 \quad \text { for } L \leqslant \ell \leqslant \frac{3}{2} L-3 \\
\frac{4}{L^{2}} \text { for } \frac{3}{2} L-2 \leqslant \ell \leqslant \frac{3}{2} L-1 \\
0 \text { for } \frac{3}{2} L \leqslant \ell \leqslant 2 L-3 \\
-\frac{2}{L^{2}} \quad \text { for } 2 L-2 \leqslant \ell \leqslant 2 L-1
\end{array}\right.
$$


giving

$$
\sum_{\ell=0}^{2 L-1}\left|\Delta^{3} h\left(\frac{\ell}{L}\right)\right|=\frac{16}{L^{2}},
$$

which satisfies (4.5) for $d=2$ with $C_{2}=16$. A similar calculation holds also for $L$ odd. Thus in both cases this choice of $h$ satisfies the conditions of Proposition 4.1.

\subsection{Higher values of $d$}

The principle used above can be extended to any value of $d$ : for sufficiently large $L$ the $(d+1)$ st finite differences of $h\left(\frac{\ell}{L}\right)$ vanish by virtue of $(4.13)$ and $(5.1)$, except at $d$ points before each breakpoint, and at each such exceptional point the absolute value of the $(d+1)$ st finite difference is a bounded multiple of $L^{-d}$. Because the number of exceptional points is bounded independently of $L$, it is then clear that (4.5) is satisfied for large $L$, completing the argument. It is only a matter of detail to construct the spline filters for particular values of $d$ greater than 2 .

Acknowledgement. I thank Hong Kong Polytechnic University, where this work was carried out, for support under its Distinguished Scholars Scheme. I also thank Hrushikesh Mhaskar and Yuguang Wang for introducing me to polynomial filters and de la ValléePoussin means respectively. The support of the Australian Research Council is gratefully acknowledged.

\section{References}

[1] H. Berens, P.L. Butzer, and S. Pawelke, Limitierungsverfahren von Reihen mehrdimensionaler Kugelfunktionen und deren Saturationsverhalten , Publ. RIMS, Kyoto University, Ser.A, 4 (1968/1969), pp. 201-268.

[2] H. Berens and L. Li, On the de la Vallée-Poussin means on the sphere, Results Math., 24 (1993), pp. $12-26$.

[3] D.L. Berman, On a class of linear operators, Doklady Akad. Nauk SSSR (N.S.), 85 (1952), pp. 13-16, (Russian).

[4] I.K. Daugavet, Some applications of the Marcinkiewicz-Berman identity, Vestnik Leningrad Univ., 23 (1968), no. 19, pp. 59-64 (1968).

[5] R.A. De Vore and G.G. Lorentz, Constructive Approximation, Springer-Verlag, Berlin, 1993.

[6] F. Filbir and W. Themistoclakis, Polynomial approximation on the sphere using scattered data, Math. Nachr., 281 (2008), pp. 650-668.

[7] E. Kogbetliantz, Recherches sur la sommabilité du séries ultrasphériques par le méthode des moyens arithmétiques, J. Math. Pures Appl. Ser. 9, 3 (1924), pp. 107-187.

[8] P.P. Korovkin, Linear Operators and Approximation Theory, Fizmatgiz, Moscow, 1959. English edition: Hindustan Publ. Co., Delhi, 1960.

[9] H.N. Mhaskar, F.J. Narcowich, J. Prestin, and J.D. Ward, Polynomial frames on the sphere, Adv. Computat. Math., 13 (2000), pp. 387-403.

[10] D.L. Ragozin, Constructive polynomial approximation on spheres and projective spaces, Trans. Amer. Math. Soc. 162 (1971), pp. 157-170.

[11] M. Reimer, A short proof of a result of Kogbetliantz on the positivity of Cesàro sums, Math. Z. 221 (1996), pp. 189-192.

[12] M. Reimer, Multivariate Polynomial Approximation, Birkhäuser Verlag, Basel, Boston, Berlin 2003. 
[13] I.H. Sloan and R.S. Womersley, Filtered hyperinterpolation - a constructive polynomial approximation on the sphere, submitted.

[14] K.P. Rustamov, On the approximation of functions on the sphere, Izv. Ross. Akad. Nauk ser. Mat, 57 (1993), no. 5, pp. 127-148. English translation in Russian Acad. Sci. Izv. Math., 43 (1994), no. 2, pp. 311-329.

[15] E.M. Stein, Interpolation in polynomial classes and Markoff's inequality, Duke Math. J., 24 (1957), pp. $467-476$.

[16] C. de la Vallée-Poussin, Leçons sur l'Approximation des Fonctions d'une Variable Réelle , GauthiersVillars, Paris 1919; 2nd edn. Chelsea Publ. Co., New York 1970.

[17] R. Yang, F. Cao, and J. Xiong, The strong converse inequality for the de la Vallée-Poussin means on the sphere, submitted. 